\title{
On the K-theoretic classification of topological phases of matter
}

\author{
Guo Chuan Thiang*† \\ University of Oxford, Mathematical Institute, Oxford, United Kingdom \\ E-mail: thgchuan@gmail.com
}

\begin{abstract}
A $K$-theoretic approach to the study of gapped topological phases was suggested by Kitaev, who produced a Periodic Table of topological insulators and superconductors, modelled on Bott periodicity. We take the algebraic viewpoint, and study gapped phases of free fermions through a twisted crossed product $C^{*}$-superalgebra associated to the symmetry data of the dynamics. We identify the $K$-theoretic difference-group of this symmetry algebra, in the sense of Karoubi, as the appropriate point of entry for $K$-theory. Thus, $K$-theory provides groups of obstructions between symmetry-compatible gapped Hamiltonians, rather than classification groups for the Hamiltonians themselves. The phenomena of periodicity and dimension-shifts in the difference-groups is shown to be a robust consequence of various isomorphisms in operator $K$-theory, which have no commutative counterpart.
\end{abstract}

Frontiers of Fundamental Physics 14

15-18 July 2014

Aix Marseille University (AMU) Saint-Charles Campus, Marseille, France

\footnotetext{
* Speaker.

${ }^{\dagger}$ The author thanks Balliol College and the Clarendon Fund for financial support. This paper is a summary of some of the results in [15].
} 


\section{Symmetry classification of quantum mechanical systems}

In a fundamental paper of Dyson [5], it was shown that quantum mechanical systems with symmetry came in three classes, corresponding to the three associative real division algebras $\mathbb{R}, \mathbb{C}$ and $\leftrightarrow$. There were actually a few "threefold ways" addressing several inequivalent classification problems, one of which was the classification of projective unitary-antiunitary representations according to their commutants. The threefold way was expanded into a tenfold way for free-fermion systems $[1,7]$ based on Cartan's ten classical compact symmetric spaces. Topology began to play a more prominent role, with the seminal paper of Kitaev [9] proposing a classification of (gapped) topological insulators and superconductors based on $K$-theory. This was a short paper, and many authors (e.g. [12]) proceeded to provide their versions of the $K$-theory classification. The paper of Freed-Moore [6] provided an account of the tenfold way based on the ten real superdivision algebras. Their subsequent analysis of quantum mechanical symmetries goes beyond the tenfold way, and they arrive at a classification scheme based on twisted equivariant $K$-theory.

Although there seems to be a consensus in the literature that the free-fermion classification problem is solved, there are in fact many inconsistencies as well as inequivalent classification problems (see $[15,14]$ for an extended discussion). In particular, conventions differ between authors, and the equivalence relations (e.g. isomorphism or homotopy) defining the classes of free-fermions are not always uniformly applied, even within the same framework. In a homotopy classification, the general intuition is that there is a topological space $Y$ of Hamiltonians (gapped or otherwise) which are compatible with certain given symmetry constraints, and homotopic Hamiltonians within this space are to be identified. Physically, homotopy corresponds to some adiabatic change of parameters, and one is only interested in capturing features of Hamiltonians which are insensitive to such operations. From this point of view, the problem is to identify $Y$ and compute its set of path-components $\pi_{0}(Y)$. This is not yet a group! Indeed, the connection to $K$-theory groups is not at all obvious. In this paper, we fill in this important gap in order to understand the nature of the $K$-theory groups appearing in Kitaev's Periodic Table - we find they do not actually classify the Hamiltonians themselves (up to homotopy), but rather the obstructions between Hamiltonians in a homotopy sense. We work with minimal assumptions and in a model-independent manner, in order not to artificially introduce topology into $Y$. Our approach is a noncommutative one, and is able to handle physically important phenomena such as the Integer Quantum Hall Effect as well as disordered systems. Thus, we are not limited to topological insulators modelled by vector bundles over Brillouin tori.

The original Periodic Table is of course Bott's table of homotopy groups of the stable classical groups [2]. Thus the answer is, in a sense, already "known". The first achievement of this paper is the identification of an appropriate question to which Bott's table (or at least the Bott periodicity of $K$-theory groups) provides the answer. The second important result is a robust proof of the dimension-shift phenomenon, which utilises results in the field of $C^{*}$-algebras and their $K$-theory. It makes clear that the noncommutative approach is not only more general, but also necessary.

\section{Basic classification principles}

Suppose $u_{t}=e^{-\mathrm{i} H t}$ is a strongly-continuous one-parameter group of unitary time evolution 
generated by a gapped Hamiltonian $H$, i.e., $0 \notin \operatorname{spec}(H)$. We assume that there is a locally compact second-countable group $G$ of dynamical symmetries, equipped with two continuous homomorphisms $\phi, \tau: G \rightarrow\{ \pm 1\}$ indicating whether the representative $g$ of $g \in G$ is unitary/antiunitary and time orientation preserving/reversing:

$$
\mathrm{gi}=\phi(g) \mathrm{ig}, \quad \mathrm{g} u_{t}=u_{\tau(g) t} \mathrm{~g} .
$$

The spectral flattening $H \mapsto \operatorname{sgn}(H)=: \Gamma$ defines a grading operator $\Gamma$. With respect to $\Gamma$, the operators g are even/odd, i.e., $\mathrm{g} \Gamma=c(g) \Gamma \mathrm{g}$, according to $c:=\phi \cdot \tau$. Wigner's Theorem says that $g \mapsto$ $\mathrm{g}$ need only be a projective unitary-antiunitary representation (PUA-rep), so there is cohomological data in the form of a Borel map $\sigma: G \times G \rightarrow \mathrm{U}(1)$ satisfying the 2-cocycle condition

$$
\sigma(x, y) \sigma(x y, z)=\sigma(y, z)^{x} \sigma(x, y z),
$$

where $\sigma(\cdot, \cdot)^{x}$ means $\sigma(\cdot, \cdot)$ if $\phi(x)=+1$ and $\overline{\sigma(\cdot, \cdot)}$ if $\phi(x)=-1$.

Thus, the symmetry constraints are abstractly specified by $(G, c, \phi, \sigma)$, and the grading operator in a concrete realisation of this data (as a $\mathbb{Z}_{2}$-graded PUA-rep) is a flattened compatible Hamiltonian. It represents the family of compatible gapped Hamiltonians $H$ which have $\operatorname{sgn}(H)=\Gamma$. More generally, $g \in G$ may act on a $C^{*}$-algebra $\mathscr{B}$ by automorphisms $\alpha_{g}$, which may be twisted by a generalised 2-cocycle. Then one is interested in graded covariant representations of the graded twisted $C^{*}$-dynamical system $(G, c, \mathscr{B}, \alpha, \sigma)$.

The covariant representation theory of a (graded) twisted dynamical system can be concisely rephrased as the ordinary (graded) representation theory of the (graded) twisted crossed product $[10,3] \mathscr{A}=\mathscr{B} \rtimes_{(\alpha, \sigma)} G$, which is a certain completion of $L^{1}(G, \mathscr{B})$ with a $(\alpha, \sigma)$-twisted convolution product. For example, a graded PUA-rep of $(G, c, \phi, \sigma)$ is a graded $*$-representation of the algebra $\mathscr{A}=\mathbb{C} \rtimes_{(\alpha, \sigma)} G$, where $\alpha_{g}$ is complex conjugation on $\mathbb{C}$ if $\phi(g)=-1$ and the identity otherwise. Note that $\mathscr{A}$ is a real algebra if $\phi$ is non-trivial. We interpret the graded algebra $\mathscr{A}$ as the symmetry algebra, generalising the notion of a group algebra. We can associate a number of algebraic objects to $\mathscr{A}$, such as its (super-)representation ring, but we will mostly be concerned with its $K$-theory groups and their interpretation in terms of homotopies of compatible Hamiltonians.

Example 2.1 ( $C T$-subgroups and the Clifford algebras). Let $A$ be a subgroup of the $C T$-group $\{1, T, C, S\}=\{ \pm 1\}^{2}$, where $T, C$ and $S=C T$ are respectively time-reversal, charge-conjugation and sublattice symmetries. The usual convention is that $T$ is antiunitary and even, $C$ is antiunitary and odd, and $S$ is unitary and odd; these determine $\phi$ and $c$. We utilise the phase freedom in the representatives $\mathrm{T}, \mathrm{C}, \mathrm{S}$ to ensure that $\mathrm{T}^{2}= \pm 1=\mathrm{C}^{2}, \mathrm{~S}^{2}=+1$, and CT $=\mathrm{TC}$; these specify the cocycle $\sigma$. Consequently, there are ten standardised choices for $(A, \sigma)$, which is a manifestation of the tenfold way. With a little work, one can show that the associated symmetry algebra $\mathbb{C} \rtimes_{(\alpha, \sigma)} A$ is, up to Morita equivalence, a complex Clifford algebra $\mathbb{C} l_{n}, n=0,1(\bmod 2)$, or a real Clifford algebra ${ }^{1} C l_{r, s}, r-s=0, \ldots, 7(\bmod 8)$. Thus there are exactly $2+8=10$ cases.

\section{3. $K$-theory and symmetry-compatible gapped Hamiltonians}

Recall that $K^{0}(X)$ of a compact Hausdorff space $X$ is the Grothendieck completion of the moniod of isomorphism classes of complex vector bundles over $X$; similarly, the operator $K_{0}(\mathscr{A})$ for a

\footnotetext{
${ }^{1}$ Our convention is that $C l_{r, s}$ has $r$ generators squaring to -1 and $s$ generators squaring to +1 .
} 
unital real or complex $C^{*}$-algebra $\mathscr{A}$ is the Grothendieck completion of the monoid of isomorphism classes of finitely-generated projective (f.g.p.) $\mathscr{A}$-modules. In the context of band insulators without additional symmetries other than a lattice $\mathbb{Z}^{d}$ of translations, the symmetry algebra is $C\left(\mathbb{T}^{d}\right)$, the continuous complex-valued functions on the Brillouin torus $\mathbb{T}^{d}$. Higher $K$-theory groups $K_{n}(\mathscr{A})$ may be defined as the $K_{0}$-group of the $n$-fold suspension of $\mathscr{A}$. However, this is a definition that only works for ungraded $\mathscr{A}$ and is difficult to interpret physically. Therefore, we turn to a model of $K$-theory due to Karoubi [8], which works for graded algebras.

Definition 3.1 (Symmetry-compatible gapped Hamiltonians). Let $(G, c, \phi, \sigma)$ be symmetry data as described in Section 2, and let $\mathscr{A}=\mathbb{C} \rtimes_{(\alpha, \sigma)} G$ be its associated symmetry algebra. Suppose $\mathscr{A}$ is unital, and let $W$ be an ungraded f.g.p. $\mathscr{A}$-module. We call $\operatorname{Grad}_{\mathscr{A}}(W)$ the set of symmetrycompatible gapped Hamiltonians on $W$. Two grading operators $\Gamma_{1}, \Gamma_{2} \in \operatorname{Grad}_{\mathscr{A}}(W)$ are said to be homotopic if there is a norm-continuous path between $\Gamma_{1}$ and $\Gamma_{2}$ within $\operatorname{Grad}_{\mathscr{A}}(W)$; in this case, we write $\Gamma_{1} \sim_{h} \Gamma_{2}$.

Note that there is a standard Banach space structure on $W$ (induced from the free module $\mathscr{A}^{n}$ which it is a direct summand of). This determines a norm topology on the bounded linear maps $W \rightarrow W$. Therefore it makes sense to talk about $\operatorname{Grad}_{\mathscr{A}}(W)$ as a topological space. By replacing $\mathbb{C}$ with some graded $C^{*}$-algebra $\mathscr{B}$ in Definition 3.1, we can also consider $\operatorname{Grad}_{\mathscr{A}}(W)$ for more general twisted crossed products $\mathscr{A}=\mathscr{B} \rtimes_{(\alpha, \sigma)} G$.

Let us now consider triples $\left(W, \Gamma_{1}, \Gamma_{2}\right)$ comprising an ungraded $\mathscr{A}$-module, and two symmetrycompatible gapped Hamiltonians $\Gamma_{i} \in \operatorname{Grad}_{\mathscr{A}}(W)$. Physically, this triple represents the ordered difference between the Hamiltonians $\Gamma_{1}$ and $\Gamma_{2}$. If $\Gamma_{1} \sim_{h} \Gamma_{2}$, we say that the triple $\left(W, \Gamma_{1}, \Gamma_{2}\right)$ is trivial. The collection of triples $\operatorname{Grad}_{\mathscr{A}}$ forms an abelian monoid under the direct sum, with the trivial triples forming a submonoid $\operatorname{Grad}_{\mathscr{A}}^{t}$.

Definition 3.2 (Difference-group of compatible Hamiltonians). Let $\mathbf{K}_{0}(\mathscr{A})$ be the quotient monoid of $\operatorname{Grad}_{\mathscr{A}}$ by the congruence generated by $\operatorname{Grad}_{\mathscr{A}}^{t}$, i.e., $\left[W, \Gamma_{1}, \Gamma_{2}\right]=\left[W^{\prime}, \Gamma_{1}^{\prime}, \Gamma_{2}^{\prime}\right]$ in $\mathbf{K}_{0}(\mathscr{A})$ if and only if there are trivial triples $\left(F, \zeta_{1}, \zeta_{2}\right)$ and $\left(F^{\prime}, \zeta_{1}^{\prime}, \zeta_{2}^{\prime}\right)$ in $\operatorname{Grad}_{\mathscr{A}}^{t}$, such that

$$
\left(W \oplus F, \Gamma_{1} \oplus \zeta_{1}, \Gamma_{2} \oplus \zeta_{2}\right)=\left(W^{\prime} \oplus F^{\prime}, \Gamma_{1}^{\prime} \oplus \zeta_{1}^{\prime}, \Gamma_{2}^{\prime} \oplus \zeta_{2}^{\prime}\right)
$$

in $\operatorname{Grad}_{\mathscr{A}}$. We call $\mathbf{K}_{0}(\mathscr{A})$ the difference-group of symmetry-compatible gapped Hamiltonians.

$\mathbf{K}_{0}(\mathscr{A})$ has very nice properties:

Theorem 3.3. $[15,8] \mathbf{K}_{0}(\mathscr{A})$ is an abelian group, with $\left[W, \Gamma_{1}, \Gamma_{2}\right]=-\left[W, \Gamma_{2}, \Gamma_{1}\right]$. Furthermore, two isomorphic triples (in the natural sense) define the same class in $\mathbf{K}_{0}(\mathscr{A})$. Also, equation $\left[W, \Gamma_{1}, \Gamma_{2}\right]+\left[W, \Gamma_{2}, \Gamma_{3}\right]=\left[W, \Gamma_{1}, \Gamma_{3}\right]$ holds in $\mathbf{K}_{0}(\mathscr{A})$. Furthermore, $\left[W, \Gamma_{1}, \Gamma_{2}\right]$ depends only on the homotopy class of $\Gamma_{i}$ in $\operatorname{Grad}_{\mathscr{A}}(W)$.

Note that the difference-group is automatically a group, where the inverse is simply the difference taken in the opposite order - there is no need for an artificial Grothendieck group construction. Differences in $\mathbf{K}_{0}(\mathscr{A})$ can be added in a "path-independent" way, and the class of a difference element has the homotopy invariance that we want from a physical perspective.

The difference group can be viewed as a generalisation of the ordinary $K_{0}$-group described in the first paragraph of this section (see Chapter III of [8] for details). For purely-even algebras $\mathscr{A}^{\mathrm{ev}}$, 
we have $\mathbf{K}_{0}\left(\mathscr{A}^{\mathrm{ev}}\right) \cong K_{0}\left(\mathscr{A}^{\mathrm{ev}}\right)$. For real graded $C^{*}$-algebras, we can define the $n$-fold "Clifford suspension" $\mathscr{A} \mapsto \mathscr{A} \hat{\otimes} C l_{0, n}$, where $C l_{0, n}$ has its natural grading. Then we can define the higher difference groups $\mathbf{K}_{n}(\mathscr{A}):=\mathbf{K}_{0}\left(\mathscr{A} \hat{\otimes} C l_{0, n}\right)$. Karoubi showed that Clifford suspension is compatible with the ordinary suspension of algebras $\mathscr{A} \mapsto C_{0}(\mathbb{R}, \mathscr{A})$, in the sense that $\mathbf{K}_{n}(\mathscr{A}) \cong \mathbf{K}_{0}\left(C_{0}\left(\mathbb{R}^{n}, \mathscr{A}\right)\right)$; in particular, $\mathbf{K}_{n}\left(\mathscr{A}^{\mathrm{ev}}\right) \cong K_{0}\left(C_{0}\left(\mathbb{R}^{n}, \mathscr{A}^{\mathrm{ev}}\right)\right)=: K_{n}\left(\mathscr{A}^{\mathrm{ev}}\right)$. Furthermore, $\mathbf{K}_{n}$ (like the ordinary $\left.K_{n}\right)$ inherits the period-8 Bott periodicity of the Clifford algebras. Similar results hold for the complex case. The upshot is that $K$-theory enters the discussion of gapped topological phases through $\mathbf{K}_{0}$, and not the ordinary ungraded $K_{0}$.

\section{Periodicities and dimension shifts in $K$-theoretic difference-groups}

Theorem 4.1 (Packer-Raeburn decomposition theorem [11]). Let $(G, c, \mathscr{B}, \alpha, \sigma)$ be a graded twisted $C^{*}$-dynamical system, and let $N$ be a closed normal subgroup of $G$ in the kernel of $c$. There is an isomorphism of graded $C^{*}$-algebras

$$
\mathscr{B} \rtimes_{(\alpha, \sigma)} G \cong\left(\mathscr{B} \rtimes_{(\alpha, \sigma)} N\right) \rtimes_{(\beta, v)} G / N,
$$

for some twisting pair $(\beta, v)$.

This is a useful result, both conceptually and computationally. As an example, we can take $G$ to be a crystallographic symmetry group, $N=\mathbb{Z}^{d}$ to be the subgroup of lattice translations, and $G / N$ as the point group. Although it may appear that twisted crossed products are complicated, they are not more complex than ordinary crossed products from the point of view of $K$-theory (which is invariant under stabilisation):

Theorem 4.2 (Packer-Raeburn stabilisation trick [11]). Let $(G, \mathscr{B}, \alpha, \sigma)$ be a twisted $C^{*}$-dynamical system, and let $\mathscr{K}$ denote the compact operators on the Hilbert space $L^{2}(G)$. There is an isomorphism

$$
\left(\mathscr{B} \rtimes_{(\alpha, \sigma)} G\right) \otimes \mathscr{K} \cong(\mathscr{B} \otimes \mathscr{K}) \rtimes_{\left(\alpha^{\prime}, 1\right)} G,
$$

for some untwisted action $\alpha^{\prime}$ of $G$ on $\mathscr{B} \otimes \mathscr{K}$.

Finally, there is a useful result in the $K$-theory of crossed products by the group $\mathbb{R}$ :

Theorem 4.3 (Connes-Thom isomorphism $[4,13])$. Let $(\mathbb{R}, \mathscr{A}, \alpha, 1)$ be an untwisted $C^{*}$-dynamical system, with $\mathscr{A}$ a real or complex (ungraded) $C^{*}$-algebra. Then $K_{n}\left(\mathscr{A} \rtimes_{(\alpha, 1)} \mathbb{R}\right) \cong K_{n-1}(\mathscr{A})$.

Iterating Theorems 4.1 and 4.2 and using Theorem 4.3, we obtain

Corollary 4.4 (Dimension shifts). Let $\left(\mathbb{R}^{d}, \mathscr{A}, \alpha, \sigma\right)$ be a twisted $C^{*}$-dynamical system. Then $K_{n}\left(\mathscr{A} \rtimes_{(\alpha, \sigma)} \mathbb{R}^{d}\right) \cong K_{n-d}(\mathscr{A})$.

Let $\tilde{\mathscr{A}}$ be the symmetry algebra for $\left(\tilde{G}, c, \mathscr{A}^{\text {ev }}, \alpha, \sigma\right)$. Suppose $\sigma$ is $\mathrm{U}(1)$-valued, and $\alpha_{g}, g \in \tilde{G}$ is either the identity or complex conjugation according to $\phi(g)= \pm 1$ (when $\phi$ is non-trivial, we assume that $\mathscr{A}^{\mathrm{ev}}$ is a complexification $\mathscr{A}_{\mathbb{R}}^{\mathrm{ev}} \otimes_{\mathbb{R}} \mathbb{C}$ ). We also assume that $\tilde{G}=\tilde{G}_{0} \times A$, where $\tilde{G}_{0}=$ $\operatorname{ker}(\alpha, c), A$ is a $C T$-subgroup, and $\sigma$ is trivial between elements of $\tilde{G}_{0}$ and $A$. Suppose $\tilde{G}_{0}$ is 
an extension of $G_{0}$ by $\mathbb{R}^{d}$, and let $\mathscr{A}_{(\mathbb{R})}^{\text {ev }}$ be the (purely-even) symmetry algebra for the subsystem $\left(G_{0}, \mathscr{A}_{(\mathbb{R})}^{\mathrm{ev}}, \alpha, \sigma\right)$.

Under these conditions, the subgroup $A$ contributes a Clifford algebra $C l_{r, s}$ or $\mathbb{C} l_{n}$ as a tensor product factor (i.e. Clifford suspension) in $\tilde{\mathscr{A}}$ (see Example 2.1). Then using Corollary 4.4, we can prove a general periodicity and dimension shift theorem:

Theorem 4.5. [15]

$$
\text { Then } \mathbf{K}_{0}(\tilde{\mathscr{A}}) \cong \begin{cases}K_{s-r-d}\left(\mathscr{A}_{\mathbb{R}}^{\mathrm{ev}}\right), & \phi \not \equiv 1, \\ K_{n-d}\left(\mathscr{A}^{\mathrm{ev}}\right), & \phi \equiv 1 .\end{cases}
$$

As an example, the algebra $\mathscr{A}^{\mathrm{ev}}=C(X)$ is often used as a model for disorder (with $X$ the disorder space). Note that the extra $\mathbb{R}^{d}$-symmetries are not assumed to enter in a trivial way, and may even be realised projectively, like the magnetic translations in the Integer Quantum Hall Effect.

\section{References}

[1] A. Altland and M. R. Zirnbauer. Nonstandard symmetry classes in mesoscopic normal-superconducting hybrid structures. Physical Review B, 55(2):1142, 1997.

[2] R. Bott. The stable homotopy of the classical groups. Annals of Mathematics, pages 313-337, 1959.

[3] R. C. Busby and H. A. Smith. Representations of twisted group algebras. Transactions of the American Mathematical Society, 149(2):503-537, 1970.

[4] A. Connes. An analogue of the Thom isomorphism for crossed products of a $C^{\star}$-algebra by an action of $\mathbb{R}$. Advances in Mathematics, 39(1):31-55, 1981.

[5] F. J. Dyson. The threefold way. Algebraic structure of symmetry groups and ensembles in quantum mechanics. Journal of Mathematical Physics, 3(6):1199-1215, 1962.

[6] D. S. Freed and G. W. Moore. Twisted equivariant matter. Annales Henri Poincaré, 14(8):1927-2023, 2013.

[7] P. Heinzner, A. Huckleberry, and M. R. Zirnbauer. Symmetry classes of disordered fermions. Communications in Mathematical Physics, 257(3):725-771, 2005.

[8] M. Karoubi. K-theory: An Introduction, volume 226 of Grundlehren der Mathematischen Wissenschaften. Springer-Verlag, Berlin, 1978.

[9] A. Kitaev. Periodic table for topological insulators and superconductors. In American Institute of Physics Conference Series, volume 1134, pages 22-30, 2009.

[10] H. Leptin. Verallgemeinerte $L^{1}$-Algebren. Mathematische Annalen, 159(1):51-76, 1965.

[11] J. A. Packer and I. Raeburn. Twisted crossed products of $C^{\star}$-algebras. Mathematical Proceedings of the Cambridge Philosophical Society, 106(02):293-311, 1989.

[12] S. Ryu, A. P. Schnyder, A. Furusaki, and A. W. Ludwig. Topological insulators and superconductors: tenfold way and dimensional hierarchy. New Journal of Physics, 12(6):065010, 2010.

[13] H. Schröder. K-theory for real $C^{\star}$-algebras and applications, volume 290 of Pitman Research Notes in Mathematics Series. Longman Scientific \& Technical, Harlow, 1993.

[14] G. C. Thiang. A note on isomorphic versus homotopic topological phases. arXiv:1412.4191, 2014.

[15] G. C. Thiang. On the $K$-theoretic classification of topological phases of matter. arXiv:1406.7366, 2014. 